DOI: $10.2478 / \mathrm{v} 10324-012-0015-2$<smiles>C1=CC2CC1C2</smiles>

VERSITA
Analele Universităţii de Vest,

Timişoara

Seria Matematică - Informatică

L, 2, (2012), 55- 66

\title{
Interchange Opportunity in Average Bandwidth Reduction in Sparse Matrices
}

\author{
Liviu Octavian Mafteiu-Scai
}

\begin{abstract}
This paper proposes two methods for determining suitable interchanges of lines/columns in case of average bandwidth reduction in sparse and symmetric matrices. First, a greedy heuristic inspired by the laws of physics (mechanics) to reduce the average bandwidth is proposed. The second method is an exact method that allows to estimate the potential of a row/column interchange to reduce the average bandwidth.
\end{abstract}

AMS Subject Classification (2000). 65F50, 65F10, 68R10, $65 \mathrm{~F} 15$

Keywords. bandwidth reduction, sparse matrix, heuristic algorithms, nature inspired heuristics

\section{Introduction}

\subsection{The Matrix Bandwidth Reduction Problem}

Reducing the bandwidth of sparse matrices is an important pre-process in solving large systems of linear equations. A proper reorganization can have a significant influence on the efficiency of a parallel implementation. The bandwidth of a matrix relates to non-zero elements position around the main diagonal and there are several ways of measuring it. The most frequently used bandwidth measure is the maximum of the absolute value of the difference 
between the row and column indices of nonzero elements, according to the relation

$$
b w=\max |i-j| ; a_{i j} \neq 0, i=\overline{1, n}, j=\overline{1, n}
$$

where $a_{i j}$ are elements of a square matrix $A$ of size $n$.

There are two types of methods to reduce the bandwidth: exact and heuristic. For exact methods, the best known works are proposed by DelCorso and Manzini [4] and Caprara and Salazar [2]. One of the first and most used heuristic is that proposed by Cuthill and McKee [3]. Other methods that use metaheuristics are: Gibbs and Poole [5], tabu search [10], genetic algorithms [7], particle swarm optimization [6], variable neighborhood search [11], ant colony optimization [12] etc. The number of heuristics algorithms proposed to solve the bandwidth reduction problem is very large and it is not easy to say which algorithm is best with respect to various problem characteristics (size, sparsity, nonzero value distribution, execution time, required memory, parallelization, etc.). Most of these approaches are based on a graph formulation. In [8] and [13] are proposed methods that does not use neither the graph formulation nor the rooted level structures but relies on applying successive transformations directly to the matrix. The bandwidth (bw) value does not provide enough information for a parallel implementation of systems equations solving. We refer here to the load balanced processor, which is a very important condition for an efficient parallelization [1]. In [8][13] is defined a new indicator, called average bandwidth which is more useful in the parallelization previously. The average bandwidth (mbw) value is:

$$
m b w=\frac{1}{m} \sum_{a_{i j} \neq 0}|i-j|
$$

where $m$ is the number of non-zero elements of the matrix $A$.

There are several reasons for using average bandwidth $(m b w)$ : - minimizing the average bandwidth has the advantage of leading to a more uniform distribution of non-zero elements around the main diagonal; - $m b w$ is more sensitive than the $b w$ to the presence around the main diagonal of the so-called "holes", that are compact regions of zero values; - $m b w$ is less sensitive to the presence of some isolated non-zero elements far from the main diagonal. A matrix which minimizes the average bandwidth will have most non-zero elements very close to the main diagonal and very few non-zero elements away from it. This is an advantage according to the paper [1], as can see in Figure $1 \mathrm{~b}$ ). An example of the above is shown in Figure 1. For the same matrix was used two algorithms CutHill-McKee for 1a) and the one proposed in [8] for 1b), first to reduce the bandwidth bw and the second to reduce the average bandwidth mbw. 


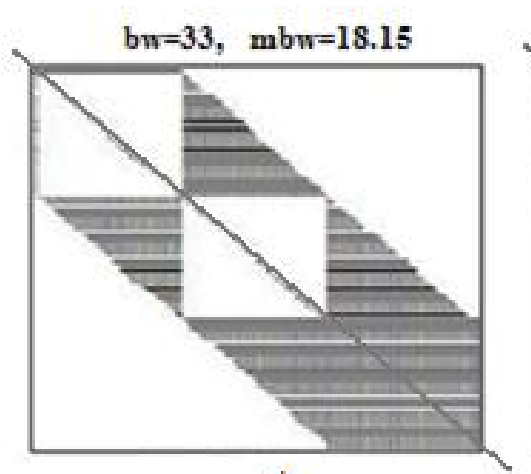

a)

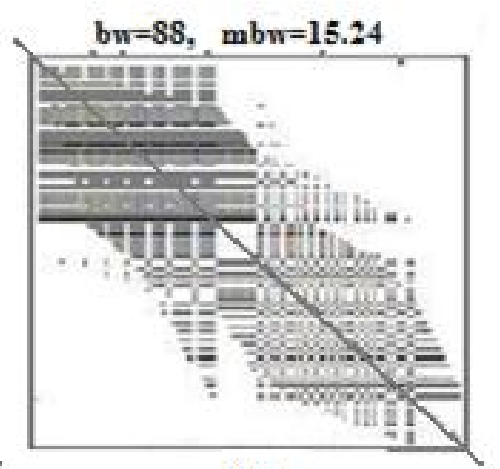

b)

Figure 1: Distributions of non-zero elements around the main diagonal

\subsection{The permutation opportunity}

The bandwidth and average bandwidth reductions consists of finding a permutation of rows and columns such that the resulting matrix has a smaller values for these. The first question that arises is if it possible to choose the most promising pair for interchange without making effectively interchange? This is not a new problem, most algorithms to reduce the bandwidth have the concern at a time finding the best permutations, or even one that ensures progress . Such concerns are described in detail in works such as [2], [3], [4], [14], [15] etc. These assessments mainly uses graph theory matrix transposition. The second question is: for a particular pair of lines/columns can be determined the opportunity of their interchange and what is the contribution (positive or negative) for average bandwidth The answer to both questions is YES and the methods are described in sections 2 and 3. Of course, this is effectively without the lines/columns interchange then be calculated from the new average bandwidth.

\section{The proposed heuristic for selection of the most promis- ing pairs}

\subsection{Physical model}

The method which we propose was inspired by the laws of physics (mechanics). The reference is the Statics chapter, precisely "the moment of a force about a point. Moment of force is the tendency of a force to rotate an object. A moment of force is valued mathematically as the product of the force and 
the moment arm: $M=F \mathrm{x} d$

The moment arm is the perpendicular distance from the point of rotation, to the line of action of the force. The line of action of a force $\mathrm{F}$ expresses the geometry of how $\mathrm{F}$ is applied. The moment of force may be thought of as a measure of the tendency of the force to cause rotation about an imaginary axis through a point.

In our case we chose as a model a bar of length $\mathrm{n}$, with support/rotation at a point called fulcrum. On this bar are put weights on both sides of the fulcrum point. Bar mass is considered equal to zero. Weights have equal masses which cause equal forces (gravitational forces). But the moments of forces are not equal, they are directly proportional to the distance weightpoint support (fulcrum).

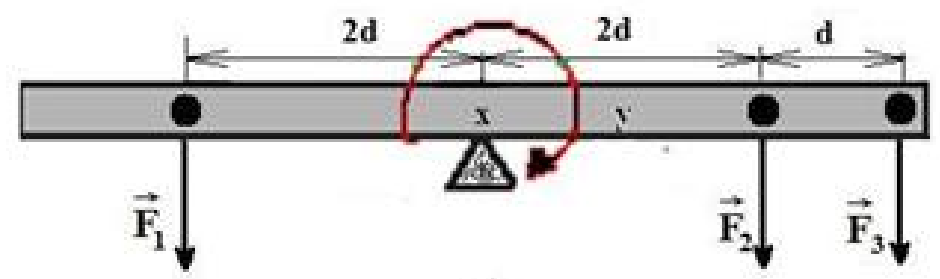

a)

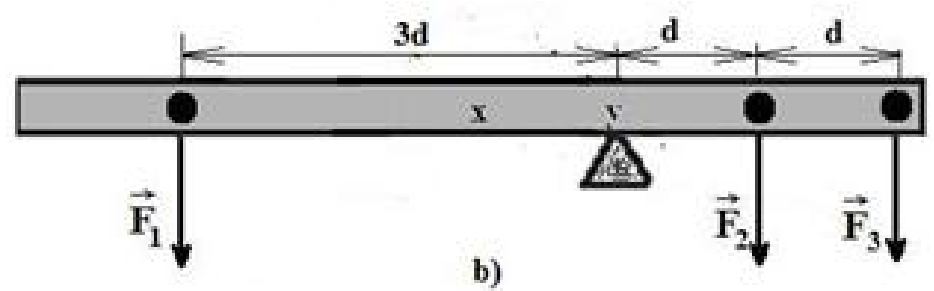

Figure 2: Illustration of the physical model

A simple example is shown in Figure 2. We consider gravitational forces $F 1=F 2=F 3=1$ and distance $d=1$. In Figure 2 a) we can see that physical system is unbalanced to the right, which is heavier, the sense of rotation being indicated by the arrow from $x$. If moments of forces are calculated separately for the left side $(M L)$ and right side $(M R)$, from the point of support $x$, are obtained $M R=2$ and $M L=5$. the resultant moment $M=M L-M R=-3$ shows disequilibrium and sense of rotation. If you want equilibrium the fulcrum must be moved to $\mathrm{y}$, for which $M L=3, M R=1+2=3$ and $M=0$. In fact, the point $y$ is the point where the center of gravity is located. From another point of view in case a) $M L+M R=7$ and in case b) $M L+M R=6$ (sum of absolute values). The 
next section will show that between these different values and reducing the average bandwidth there is a direct connection. $Y$ point coordinates, which provides equilibrium balance is trivial to calculate and is not insisted on it now.

\subsection{The analogy between the physical model and matrix}

The basic idea is: a line matrix is to consider as a balance, the fulcrum is at lines junction with the main diagonal and non-zero values are weights (forces) on the arms of the same mass balance, with mass equal to 1 . Weights are at different distances from the center (column index) of bar, which are equal to 1. On each line (bar) can be found $n$ non-zero values (weights) at a distance between 1 and n-1 between them, integer values.

Physically, great distance from the fulcrum involves a great moment of force and vice versa. As was mentioned in the previous section, between absolute value of the resulting moment and average bandwidth there is a direct correspondence, ie M largest for a line represents a significant contribution (balance) to the average bandwidth and vice versa.

Balance is more stable if center of gravity is closer to the fulcrum. In term of matrix transposition this measn that the center of gravity must be closer or to coincide (ideal case) with line intersection with main diagonal. Consequently, the optimization in this respect would mean moving the fulcrum point toward the heavier. Because we can't move the diagonal, the solution is to move the line (up or down) which is equivalent to changing the point of intersection with the main diagonal. Because only a single line transfer is not allowed (mathematically would mean changing the system of equations), the only chance is to find another balance/line whose center of gravity to be the other side of the main diagonal in relation to the first balance/line.

Interchanging the positions of the two balances/lines would improve the state of equilibrium of the entire system / matrix. That's actually a interchange of two lines and the effect would be a lower average bandwidth.

The entire matrix is regarded as a system of balances (lines) mounted on a single axle (main diagonal). It is intuitive that a state of the system as close to general equilibrium has resulted in a lower value of the average bandwidth.

A simplified example can be seen in Figure 3 that represenst the initial state of the physical model. In this example $\mathrm{i}=4, \mathrm{j}=8, \mathrm{k}=13$ (numbering line/columns is from 0 to $\mathrm{n}-1$ for a matrix of size $\mathrm{n}=17$ )

For an easy method understanding we introduce the following measures/indicators for lines of matrix: left moment of forces $(M L)$, right moment of forces $(M R)$, the resultant moment $(M)$, center of gravity for a line $(C G)$, optimization 


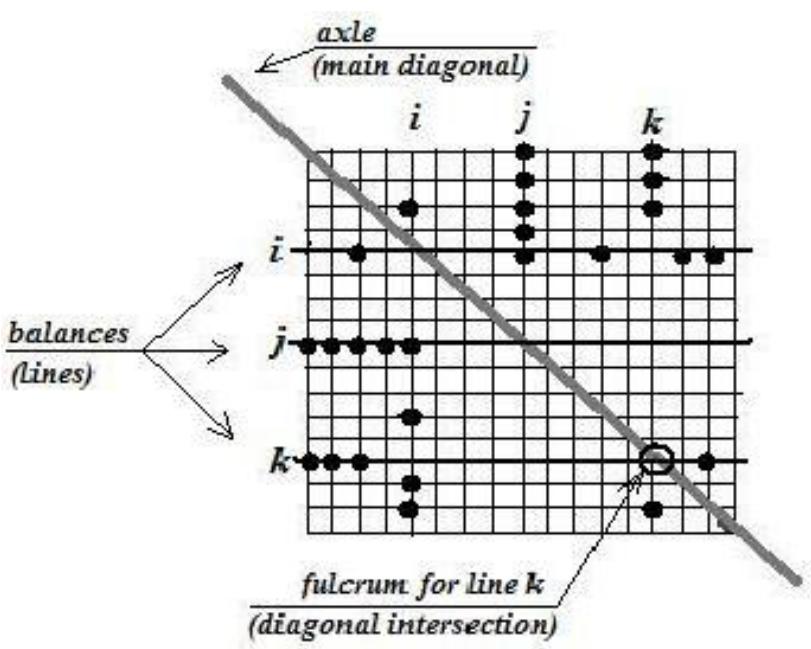

Figure 3: The analogy between the physical model and matrix: initial state

interval $(O I)$ and sense of optimization $(S O)$.

Calculation relation of $C G_{i}$ is:

$$
C G_{i}=\frac{1}{m} \sum_{j=1}^{n} a_{i j} j, a_{i j} \neq 0
$$

where $i$ is line index, $j$ is column index, $n$ is the matrix dimension and $m$ is the number of non-zero values of line $i$.

$C G i$ interpretation: the value is the physical point of support (new fulcrum) for which this balance is in equilibrium. In terms of value $m b w$, if the line is translated to position $C G i$ will have an optimization of $m b w$. The value $C G i$ shows the optimal positioning of a line in relation to the main diagonal for a minimum contribution of this line to the $m b w$. In reality, we need a permutation lines and the effect on $m b w$ also depends on the moving the line located at position $C G i$ to position $i$, a matter discussed later. Because $C G i$ values obtained are real numbers, in practice these values are recommended to be rounded to the nearest integer value.

The relations for calculation $M L i, M R i$ and $M$ are:

$$
\begin{aligned}
M L_{i} & =\sum_{j=1}^{i-1} a_{i j}(i-j), a_{i j} \neq 0 \\
M R_{i} & =\sum_{j=i+1}^{n} a_{i j}(j-i), a_{i j} \neq 0
\end{aligned}
$$




$$
M_{i}=M L_{i}-M R_{i}
$$

The optimization interval $O I_{i}$ for line $i$ is the interval located between $i$ and $C G_{i}$.

$$
O I_{i}= \begin{cases}\left(i, C G_{i}\right] & \text { if } C G i>i \\ \left(C G_{i}, i\right) & \text { if } C G i<i \\ \text { null } & \text { otherwise }\end{cases}
$$

The sign of optimization is:

$$
S O_{i}= \begin{cases}+ & \text { if }(i-C G i)<0 \\ - & \text { if }(i-C G i)>0\end{cases}
$$

Remark: relation (8) is not necessary, because $S O i$ is the sign of the value Mi obtained by relation (6).

Thus, for a permutation $(i, j)$ to generate a reduction in $m b w$, conditions must be fulfilled:

$$
-O I_{i} \cap O I_{j} \neq \emptyset
$$

- opposite signs for $S O i$ and $S O j$

For a global picture of matrix, in terms of pairs that provide a larger decrease in the value of $m b w$, are preferred lines with highest values of $M$, in correlation by previous conditions.

In Figure 4 are given forms of three matrices resulting from the matrix shown in Figure 3, after performing three possible interchanges of lines / columns. As can be seen in Figure 3, Figure 4 and Table 1, there are good interchanges (e.g. $(4,13),(4,8))$ and is not optimal the interchange $(8,4)$ in terms of average bandwidth mbw. In Figure 3 and 4 dots represent non-zero values for these three lines represented.

In accordance to selection criteria and conditions set out above, pairs that provide the greatest decrease in mbw are: $(4,13),(4,8)$ and $(1,8)$. Permutation (8.13) increases $m b w$ and the unfavorable permutation is (8.16).

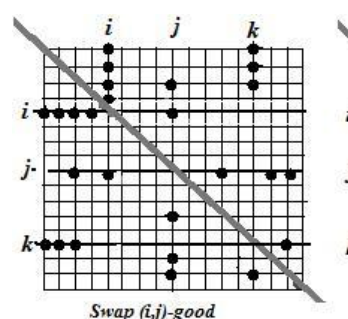

Swap (i,j)-good

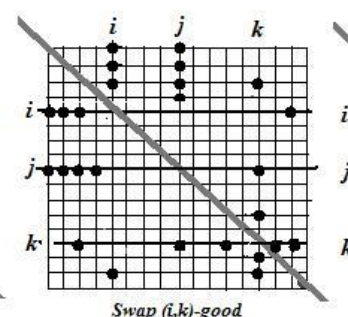

Swap $(i, k)$-good

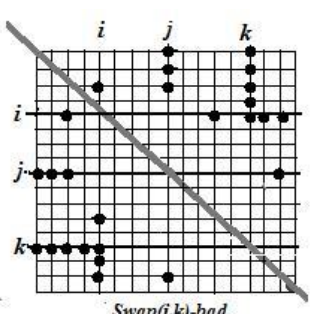

Swap $(, k)$-bad

Figure 4: Matrix from Figure 3 after interchanges $(4,8),(4,13)$ and $(8,13)$

For processing and analysis, a greedy approach was used. Sort have been 
Table 1: Examples of interchanges

\begin{tabular}{llll}
\hline Line index & M & OI & SO \\
\hline 0 & -21 & $(0,7.00]$ & - \\
\hline 1 & -19 & $(1,7.33]$ & - \\
\hline 2 & -19 & $(2,6.75]$ & - \\
\hline 3 & -5 & $(3,5.50]$ & - \\
\hline $\mathbf{4}$ & $\mathbf{- 3 0}$ & $(\mathbf{4}, \mathbf{9 . 0 0}]$ & - \\
\hline 5 & 0 & & \\
\hline 6 & 0 & & \\
\hline 7 & 0 & & \\
\hline $\mathbf{8}$ & $\mathbf{3 0}$ & {$[\mathbf{3}, \mathbf{8})$} & + \\
\hline 9 & 0 & & \\
\hline 10 & 0 & & \\
\hline 11 & 7 & {$[7.50,11)$} & + \\
\hline 12 & 0 & & \\
\hline $\mathbf{1 3}$ & $\mathbf{3 4}$ & {$[\mathbf{6 . 2 0}, \mathbf{1 3})$} & + \\
\hline 14 & 10 & {$[9.00,14)$} & + \\
\hline 15 & 13 & {$[10.67,15)$} & + \\
\hline 16 & 0 & & \\
\hline \multicolumn{5}{c}{} \\
\hline
\end{tabular}

made for the moments of lines values. For now, only qualitative results are provided after processing. A further analysis in terms of providing quantitative values is desirable.

Finally, the algorithm determines sets of pairs of interchange lines/columns which can lead to optimization.

\section{Qualitative and quantitative measurement of oppor- tunity for interchange.}

The proposed method determines the opportunity for interchange of two lines/columns to reduce mbw value. We also calculated how much increase/decrease mbw value and its new value after interchange. There is no needto lines/columns swap and then calculate the new mbw. In our method the computing effort is considerably less. For an easier understanding we will use the example presented in Figure 5.

For the given matrix, we want to know how and how much is it affected the 


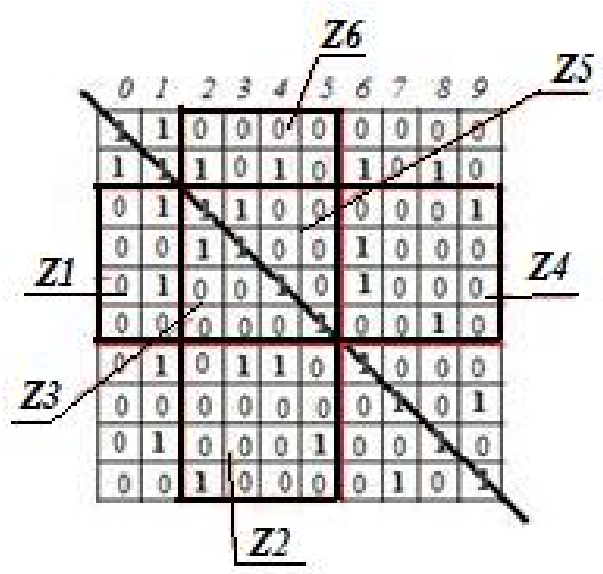

Figure 5: Matrix and areas generated by lines/columns 2 and 5

average bandwidth mbw if desired swap lines/columns is 2 and 5, without making interchange $(2,5)$ and recalculation the matrix average bandwidth. Lines and columns 2 and 5 cause six areas, equal in pairs: Z1-Z6, Z2-Z3, $Z 4-Z 5$ respectively. In determining the proposed indicators (qualitative and quantitative) is sufficient the analysis and processing areas $Z 1, Z 3$ and $Z 4$ (or Z2, Z5, Z6).

In general case of a matrix $A$, for two lines $L_{i}$ and $L_{j}(i<j)$ which can be interchanged, our algorithm follow the relation (9) to determine the contribution to the average bandwidth. $S(k)$ is the contribution of a pair of corresponding matrix elements, ie elements which are interchanged $A_{i k}$ and $A_{j k}$, where $i$ and $j$ are indices of the line and $k$ is the index of the column.

$$
S(k)= \begin{cases}\left(A\left[L_{j}\right][k]-A\left[L_{i}\right][k]\right)\left(L_{j}-L_{i}\right) & \text { in Z1 } \\ \left(A\left[L_{i}\right][k]-A\left[L_{j}\right][k]\right)\left(L_{j}-L_{i}\right) & \text { in Z4 } \\ \left(A\left[L_{i}\right][k]-A\left[L_{j}\right][k]\right)\left(2 k-L_{i}-L_{j}\right) & \text { in Z3 }\end{cases}
$$

$S(k)$ value shows how many units contribute (positively or negatively) to the average bandwidth the swap of $A_{i k}$ and $A_{j k}$ elements. Positive values indicate an optimization and a negative value indicate a reduction in quality. Relation (10) shows the overall value for swap $(i, j)$, ie for all columns of the two lines. The sign of $\Delta$ indicator shows the quality of interchange $(i, j)$ : positive means GOOD and negative means BAD. Positive sign shows an optimization, ie a decrease in the average bandwidth, and the negative sign shows an increase in the average bandwidth. Null values show that the average bandwidth remains unchanged. Numerical value of $\Delta$ shows the total 
Table 2: A few examples from the analysis of the matrix from Figure 4

\begin{tabular}{lllllllll}
\hline Interchange pair & $(5,9)$ & $(5,6)$ & $(2,6)$ & $(5,8)$ & $(5,7)$ & $(2,5)$ & $(7,0)$ & $(2,9)$ \\
\hline$\Delta$ sign & + & + & + & + & null & - & - & - \\
\hline Effect on mbw & $\downarrow$ & $\downarrow$ & $\downarrow$ & $\downarrow$ & $=$ & $\uparrow$ & $\uparrow$ & $\uparrow$ \\
\hline$\Delta$ value & 6 & 4 & 4 & 3 & 0 & 4 & 12 & 15 \\
\hline New mbw & 2.63 & 2.81 & 2.81 & 2.90 & 3.18 & 3.54 & 4.27 & 4.54 \\
\hline
\end{tabular}

Table 3: A few examples from the analysis of the matrix from Figure 5

\begin{tabular}{llllllll}
\hline Interchange pair & $(4,13)$ & $(4,8)$ & $(1,8)$ & $(2,8)$ & $(8,13)$ & $(8,15)$ & $(8,16)$ \\
\hline$\Delta$ value & 31 & 24 & 23 & 22 & 15 & 31 & 40 \\
\hline$\Delta$ sign & + & + & + & + & - & - & - \\
\hline
\end{tabular}

contribution (positive, negative or zero) on average bandwidth.

$$
\Delta=\sum_{k=1}^{n} S(k)
$$

where $n$ is the matrix dimension.

If you want to determine the new value of the average bandwidth after interchange can use relation (11). Note that there is no need for a global computing of the average bandwidth with relation (2).

$$
m b w=m b w_{0}-\frac{\Delta}{m^{\prime}}
$$

where $m$ ' denotes the number of non-zero elements of the matrix $A$, under the main diagonal. The $m$ ' is only $m / 2$ from relation (2) because we work only under main diagonal in this case. The $m b w_{0}$ is the initial value for average bandwidth in case of matrix $A$.

Some examples for the matrix shown in Figure 4 are presented in Table 2. The dimension for matrix is 10, number of non-zero values is $11(22 / 2)$ and the value of initial average bandwidth $m b w_{0}$ is 3.18 .

Note: The proposed approach also allows pre-computing bandwidth value bw, with much less computing effort than using relation (1) or similar to the relations $(9,10,11)$. Ultimately, it is a simply problem of $\min / \max$, in which are known line-column indexes of non-zero elements of where they go after the interchange. But this is not the subject of this paper.

Table 3 contains some results (sorted descending) for matrix exemplified in Figure 5. 


\section{Conclusion and future work}

These two approaches support the methods proposed in [8] and [13], where the problem of reducing bandwidth and average bandwidth is through direct processing of the matrix, without using concepts from graph theory.

In terms of complexity and calculation time, the approaches are simple and fast, especially if they are combined.

The heuristic method, inspired from nature (law of physics) presented in section 2 provide only qualitative results after processing. A further analysis in terms of providing quantitative values is desirable.

Combining these two methods is a good way to choose the pair/pairs that provide an optimum in reducing mbw. As a way of working, first, apply heuristic presented in paragraph 1 for finding the best pair in order to reduce mbw. Then, only for these pairs the exact method is applied and sorting them according to their effectiveness.

Combining these two methods together with techniques like genetic, swarm and ACO it is the present and future concern for us in order to obtain an efficient algorithm for reducing average bandwidth.

A parallelization of these methods is desirable to quickly obtain the global optimum in terms of the best permutations of rows / columns at a time.

\section{References}

[1] P. Arbenz, A. Cleary, J. Dongarra, and Heglan. M., Parallel numerical linear algebra.chapter A comparison of parallel solvers for diagonally dominant and general narrowbanded linear systems, Nova Science Publishers, Inc., Commack, NY, USA, 2001

[2] A. Caprara and J. Salazar-Gonzalez, Laying out sparse graphs with provably minimum bandwidth, INFORMS J. on Computing, 17/3, (2005), 356-373.

[3] E. Cuthill and J. McKee, Reducing the bandwidth of sparse symmetric matrices, Proc. of ACM, (1969), 157-172.

[4] G. M. Del Corso and G. Manzini, Finding exact solutions to the bandwidth minimization problem, Computing, (1999), 189-203.

[5] N. E. Gibbs, W. G. Poole, and P. K. Stockmeyer, An algorithm for reducing the bandwidth and profile of a sparse matrix, SIAM Journal on Numerical Analysis, (1976), 236-250.

[6] A. Lim, J. Lin, and F. Xiao, Particle swarm optimization and hill climbing for the bandwidth minimization problem, Applied Intelligence, 26/3, (2007), 175-182.

[7] A. Lim, B. Rodrigues, and F. Xiao, Heuristics for matrix bandwidth reduction, European Journal of Operational Research, 174/1, (2006), 69-91. 
[8] L. O. Mafteiu-Scai, Bandwidth reduction on sparse matrix, West University of Timisoara Annals, XLVIII/3, (2010.)

[9] R. Marti, V. Campos, and E. Pinana, A branch and bound algorithm for the matrix bandwidth minimization, European Journal of Operational Research, 186, (2008), 513-528.

[10] R. Marti, M. Laguna, F. Glover, and V. Campos, Reducing the bandwidth of a sparse matrix with tabu search, European Journal of Operational Research, 135, (2001), 450-459.

[11] N. Mladenovic, D. Urosevic, D. Perez-Brito, and C. Garcia-Gonzalez, Variable neighbourhood search for bandwidth reduction, European Journal of Operational Research, 200, (2010), 14-27.

[12] C. Pintea, G. Crisan, and C. Chira, A Hybrid ACO Approach to the Matrix Bandwidth Minimization Problem, M. Graa Romay et al., Springer-Verlag Berlin Heidelberg, 2010

[13] L. O. Mafteiu-Scai, V. Negru, D. Zaharie, and O. Aritoni, Average bandwidth reduction in sparse matrices using hybrid heuristics, Studia Universitatis Babes-Bolyai University, Cluj Napoca, 3/2011, (2011.)

[14] R. Mart, V. Campos, and E. Piana, A Branch and Bound Algorithm for the Matrix Bandwidth Minimization, European Journal of Operational Research 186, (2010), $513-528$.

[15] Eduardo Rodriguez-Tello, Jin-Kao Hao, and Jose Torres-Jimenez, An Improved Simulated Annealing Algorithm for Bandwidth Minimization, European Journal of Operational Research, 185(3): 1319-1335, Elsevier, (2008.)

Liviu Octavian Mafteiu-Scai

Department of Computer Science

University of Timisoara

V. Parvan nr. 4

Timisoara

Romania

E-mail: lscai@mail.info.uvt.ro

Received: 10.01 .2012

Accepted: 15.10 .2012

Revised: 10.09.2012 